\title{
Limited retromuscular fibrofatty tissue resection in upper blepharoplasty for senile patients
}

\author{
Seo Gil Choi, Sang Woo Suk, \\ Kun Chul Yoon \\ Department of Plastic and Reconstructive \\ Surgery, Kangbuk Samsung Hospital, \\ Sungkyunkwan University School of \\ Medicine, Seoul, Korea
}

\begin{abstract}
Background Studies of eyelid anatomy suggest an absence of supratarsal folds and a redundancy of retromuscular tissue in many Asians. Aggressive retromuscular tissue resection during senile blepharoplasty can lead to complications such as hematoma and extra fold formation. A thin fold is considered aesthetically pleasing, but upper eyelid hollowness is undesirable. Therefore, senile blepharoplasty allows relatively little room for retromuscular resection.

Methods Between January 2016 and March 2019, blepharoplasty without a brow procedure was performed in 48 patients aged 55 years and older. In some patients with thin eyelids, the upper retromuscular tissue was explored, and the surgeon decided intraoperatively whether to perform resection. In total, 36 of 48 patients underwent retromuscular tissue resection, which was confined to the central and lateral aspects of the eyelid within 7-10 $\mathrm{mm}$ from the incision in the superior direction to create a crisp, thin fold without hollowness. The skin was closed, with the closure including the levator palpebrae superioris (levator) muscle, to create a weak, natural-looking, and dynamic fold instead of the buried dermo-levator suture typically created during double eyelid surgery.

Results In the 72 eyelids that underwent resection, hematoma or extra fold formation was absent. In all cases, thin folds were achieved without an increase in the hollowness of the upper eyelid. Most patients were satisfied with their natural-looking postoperative appearance.

Conclusions When indicated, limited retromuscular resection is recommended in cases of senile blepharoplasty. The resection should be confined to the central and the lateral aspects of the eyelid, within 7-10 $\mathrm{mm}$ in the superior direction from the incision, to create a crisp thin fold without hollowness.
\end{abstract}

Keywords Eyelids / Blepharoplasty / Orbit evisceration

\section{INTRODUCTION}

Double eyelid surgery and senile blepharoplasty for rejuvenation are considered similar procedures.

Received: Aug 5, 2019 Revised: Sep 16, 2019 Accepted: Sep 19, 2019 Correspondence: Kun Chul Yoon Department of Plastic and Reconstructive Surgery, Kangbuk Samsung Hospital, Sungkyunkwan University School of Medicine, 29 Saemunan-ro, Jongno-gu, Seoul 03181, Korea Tel: +82-2-2001-2178, Fax: +82-2-2001-2177, E-mail: kcyoon548@gmail. com

Copyright @ 2019 The Korean Society for Aesthetic Plastic Surgery.

This is an Open Access article distributed under the terms of the Creative Commons Attribution Non-Commercial License (http://creativecommons.org/licenses/by-nc/4.0/) which permits unrestricted non-commercial use, distribution, and reproduction in any medium, provided the original work is properly cited. $\quad$ www.e-aaps.org
Double eyelid surgery, more correctly termed blepharoplasty for young Asians, involves creating a fold to achieve a beautiful, natural-looking eyelid. In contrast, senile blepharoplasty involves rejuvenating the eyelids by resecting the sagging tissue and creating folds.

Yoon and Park [1] classified the resectable tissues in the upper eyelid as peri-incisional tissue, orbital fat, pretarsal tissue, and retromuscular fat. In their study on young Asian patients, severe puffiness was observed in $40 \%$ of cases, and all resectable tissue was appropriately removed to achieve a natural-looking, beautiful, and thin eyelid.

Resection that is performed to obtain a thin eyelid in cases of senile blepharoplasty is not the same as that performed for young individuals, since with age, tissue loses its elasticity due to degenera- 
tive changes. Aggressive and wide removal of retromuscular tissue and orbital fat can lead to hematoma, extra fold formation, and an undesired hollowness, which is very different from the intended outcome of youthful eyelids. Conversely, if retromuscular tissue removal is not conducted, the results are mediocre because an artificial-looking, thick fold is obtained, as shown in Figs. 1 and 2.

During senile blepharoplasty, the purpose of retromuscular tissue resection is not to make the entire eyelid thin, but rather to create a thinned natural fold. To achieve this, we confined the retromuscular tissue resection to the area to be folded after the fold is created: the central and lateral aspect of the eyelid within 7-10 mm in the superior direction from the incision.
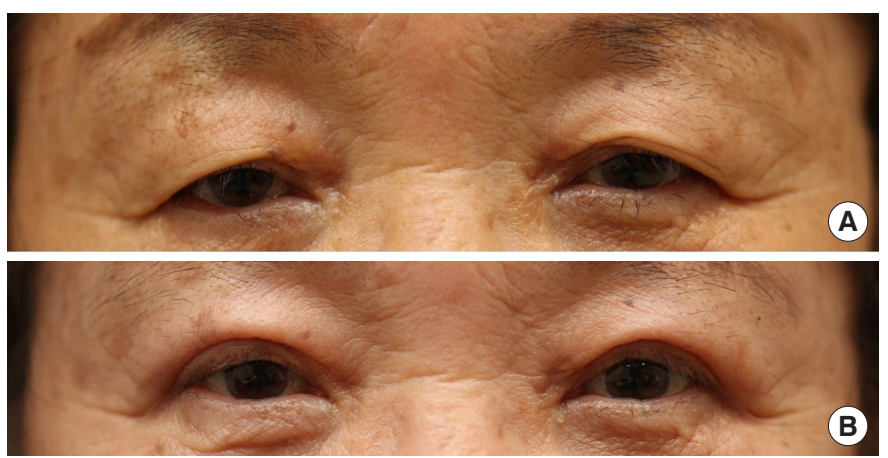

Fig. 1. (A) A 70-year-old female patient had previously undergone upper blepharoplasty without retromuscular fibro-adipose tissue resection. (B) She presented to us 4 months postoperatively with undesired puffy upper eyelid folds.

\section{METHODS}

This study was approved by the Institutional Review Board of our institution (IRB No. 2019-07-020) and was performed in accordance with the principles of the Declaration of Helsinki. Informed consent was obtained from all patients.

A total of 48 patients (13 male and 35 female) with an average age of 64.5 years (range, 55-83 years) who underwent upper blepharoplasty surgery without brow procedures between January 2016 and March 2019 were included in this study. Patients who underwent brow procedures were excluded. All conventional eyelid blepharoplasty procedures were performed by the same surgeon (KCY) for rejuvenation and correction of blepharoptosis. Of the 96 eyelids in the study, limited retromuscular tissue resection was indicated in 72 eyelids (from 36 patients), and 24 eyelids (24\%) had some degree of senile blepharoptosis and underwent levator procedures (Table 1).

\section{Indications for retromuscular tissue resection}

It is difficult to preoperatively evaluate the exact amount of retromuscular tissue present. Therefore, retromuscular tissue removal was performed intraoperatively in patients who presented with excessive fatty tissue beneath the muscle. Even if resection of retromuscular tissue was not planned for the patient before surgery, it was performed intraoperatively if a considerable amount of tissue was found after dissection.

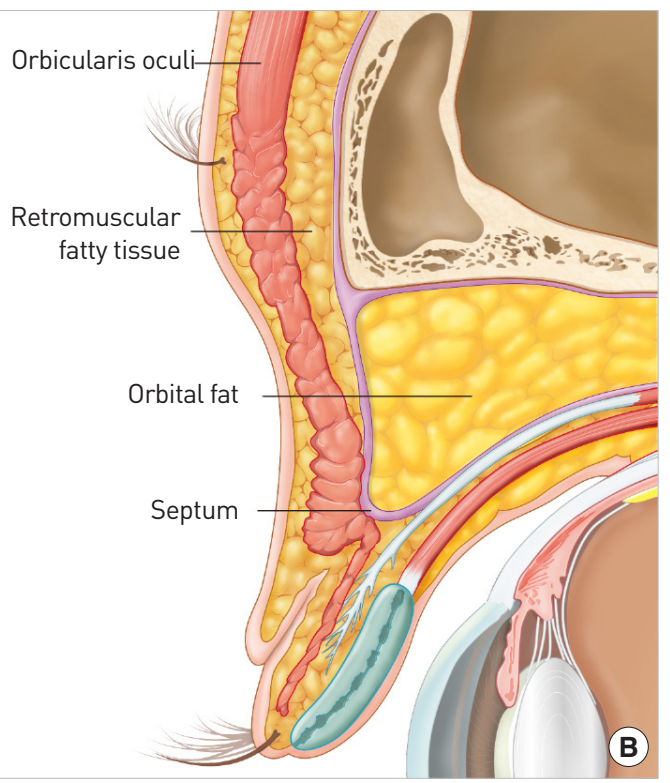

Fig. 2. (A) A thick fold is made without resection of retromuscular fat tissue. (B) A thin, pliable, and natural-looking fold is made after limited resection of retromuscular fat tissue. 


\section{Surgical technique}

\section{Peri-incisional tissue removal}

We designed a skin removal procedure as shown in Fig. 3 and infiltrated $1 \mathrm{~mL}$ of $2 \%$ lidocaine with 1:100,000 epinephrine. We then excised the skin, as well as a strip of orbicularis oculi muscle (OOM) along the lower margin of the skin excision.

\section{Retromuscular tissue exploration}

We opened the orbital septum from the lateral to the medial direction after carefully identifying the preaponeurotic fat. We then hooked the upper rim of the OOM and identified the retromuscular fatty tissue beneath the OOM. We carefully resected the retromuscular tissue from the lateral to the medial direction, while not violating the muscle fascia except in the nasal third of the eyelid, within ap-

Table 1. Patient characteristics ( $n=96)$

\begin{tabular}{lc}
\hline Characteristic & Eyelids, No. (\%) \\
\hline Sex & $26(27.0)$ \\
Male & $70(73.0)$ \\
Female & \\
Retromuscular tissue & $72(75.0)$ \\
Resection & $24(25.0)$ \\
No resection & \\
Preoperative evaluation & $72(75.0)$ \\
Pseudo-blepharoptosis & $24(25.0)$ \\
True senile blepharoptosis (unilateral or bilateral) & \\
\hline
\end{tabular}

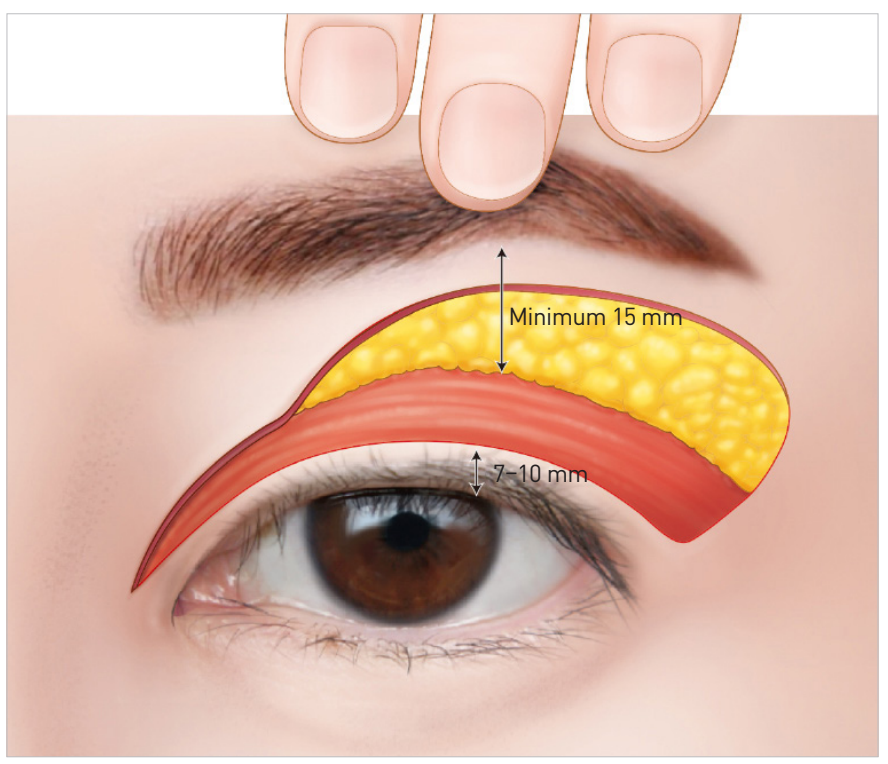

Fig. 3. Schematic presentation of the incision line and the location of limited retromuscular tissue resection (the central and the lateral aspect of the eyelid within $7-10 \mathrm{~mm}$ in the superior direction from the incision). proximately $7-10 \mathrm{~mm}$ in the superior direction from the rim of the incision (Fig. 3).

\section{Orbital fat excision}

Next, we excised the protruding nasal orbital fat, and the central and lateral orbital fat overflowing over the tarsus was removed using electrocautery. We then repaired or advanced the levator muscle in the eyelid that displayed blepharoptosis.

\section{Creation of a fold using skin closure}

We applied several skin closure sutures, including in the posterior lamella of the eyelid. At this time, we incorporated the free edge of the orbital fat and fascia (Fig. 4) to prevent unwanted adhesions (extra folds).

\section{Evaluation of patient satisfaction}

Among 36 patients who underwent upper blepharoplasty with retromuscular tissue resection, 31 patients were followed up for $>6$ months postoperatively and were interviewed about their degree of satisfaction with the results of the procedure (Table 2).

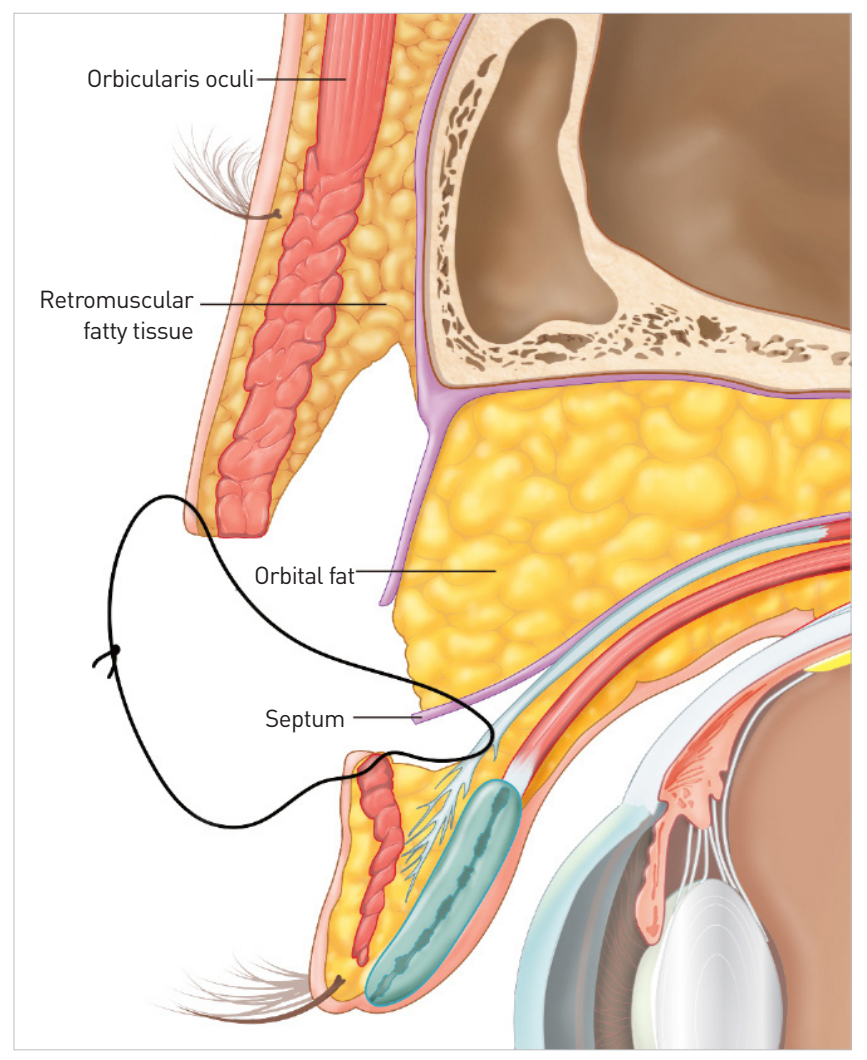

Fig. 4. After confirming complete hemostasis, the skin, orbicularis oculi muscle, orbital fat, and levator aponeurosis were sutured together to create a double eyelid fold at approximately five sites using Nylon 6-0 sutures. 
Table 2. Degree of satisfaction $(n=31)$

\begin{tabular}{lc}
\hline Rating & No. of patients $(\%)$ \\
\hline Excellent & $23(74.2)$ \\
Good & $5(16.1)$ \\
Moderate & $2(6.5)$ \\
Dissatisfied & $1(3.2)$ \\
Total patients & $31(100)$ \\
\hline
\end{tabular}
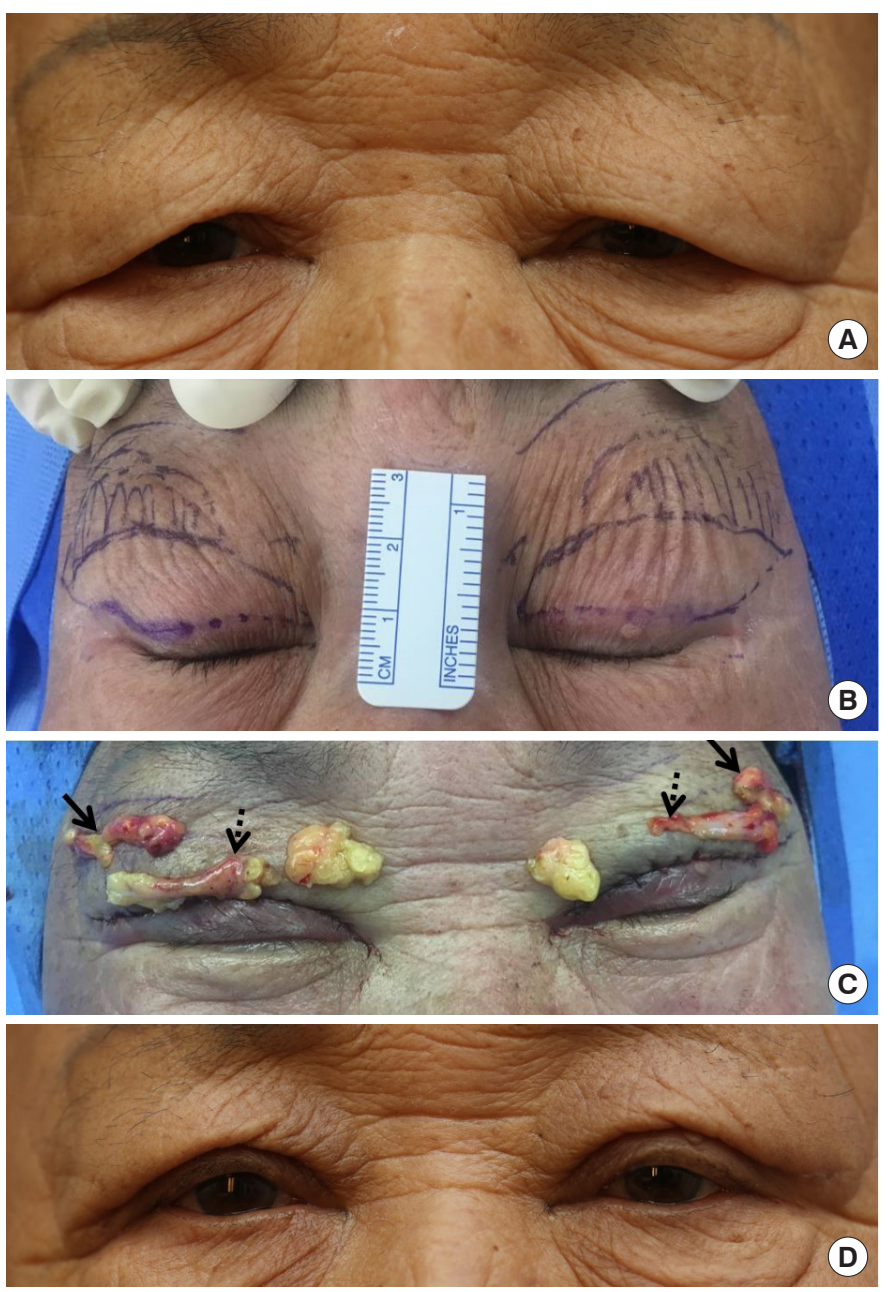

Fig. 5. Case of a 77-year-old female patient. (A) Preoperative photo. (B) The skin incision line and the retromuscular tissue resection boundary (slash-filled area). (C) Resection of retromuscular tissue (black arrow) and central orbital fat (dotted arrow). (D) Postoperative photo taken 1 month after the procedure.

\section{RESULTS}

We observed no cases of postoperative complications of tissue resection, such as hollowness, hematoma, or extra fold formation. The majority of the patients reported good to excellent satisfaction

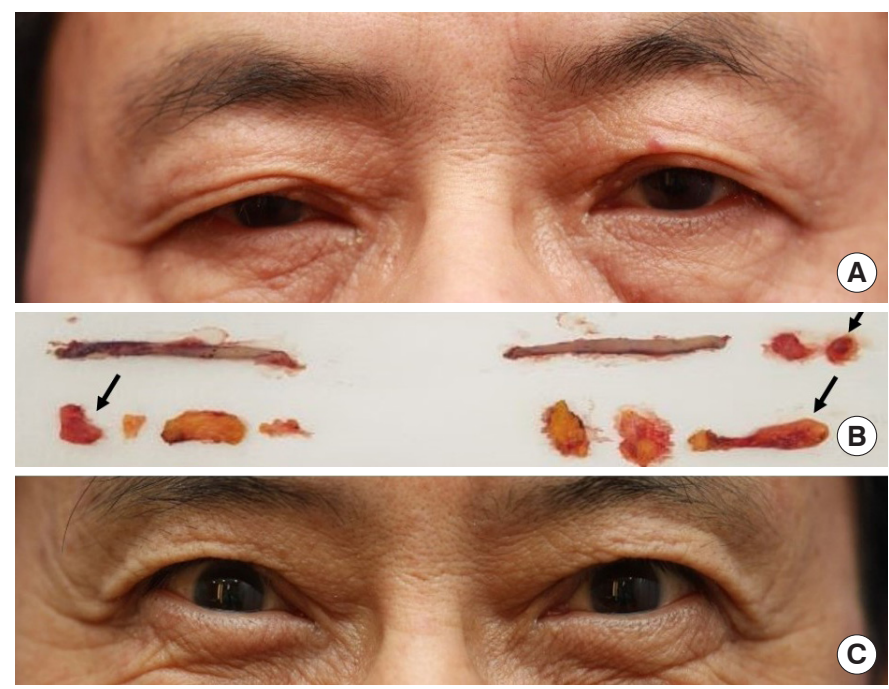

Fig. 6. Case of a 64-year-old male patient. (A) Preoperative photo. (B) Resected tissue; the black arrow points to the resected retromuscular fat. (C) Postoperative photo taken 5 months after the procedure.

and did not feel that the fold looked artificial. Of the 31 patients, 28 (90.2\%) were satisfied, with a reported result of "excellent" or "good." The remaining three patients wanted a more prominent pretarsus (a larger fold), either bilaterally or unilaterally, and underwent minor revision.

\section{Case 1}

In a 77-year-old woman with severe pseudoptosis, upper blepharoplasty was performed with limited retromuscular tissue removal and resection of the skin, the OOM, and the medial and the central orbital fat pads (Fig. 5). By 1 month after limited retromuscular tissue removal, thin, pliable, natural-looking folds had been achieved.

\section{Case 2}

In a 64-year-old man with mild acquired bilateral blepharoptosis, upper blepharoplasty was performed with limited retromuscular tissue removal and resection of the skin, the OOM, and the medial and the lateral orbital fat pads in conjunction with levator repair (Fig. 6). By 6 months after limited retromuscular tissue removal, thin, pliable, natural-looking folds had been achieved.

\section{Case 3}

In a 68-year-old woman with bilateral pseudoptosis, upper blepharoplasty was performed (Fig. 7). By 7 months after limited retromuscular resection, thin, pliable, natural-looking folds had been achieved.

\section{Case 4}

In a 54-year-old woman with bilateral pseudoptosis, upper blepharoplasty with limited retromuscular resection was performed (Fig. 8). Due to skin laxity, the patient underwent revisional surgery on 

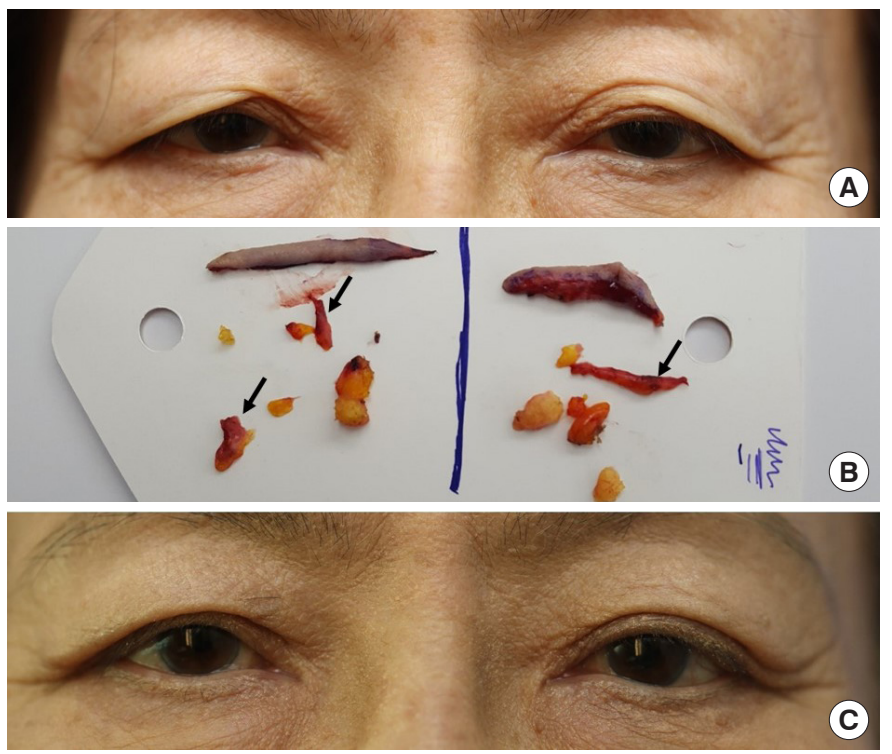

Fig. 7. Case of a 68-year-old female patient. (A) Preoperative photo. (B) Resected tissue; the black arrow points to the resected retromuscular fat. (C) Postoperative photo taken 7 months after the procedure.

the right upper eyelid 6 months after the primary operation. Excision of the remnant skin and a small amount of fat tissue removal were performed in revisional surgery.

\section{DISCUSSION}

The first tissue removed during occidental blepharoplasty was the periorbital fat. Bourguet [2] reported the presence of separate fat compartments in the eyelids and described his technique for excising excess fat. Modern blepharoplasty, as well as the detailed anatomy of the orbital fat compartments, was described by Castanares in 1951 [3]. The fatty tissues of the upper eyelid consist of the orbital fat pad in the medial, central, and lateral parts and the retromuscular fat pad in the superolateral part [4]. Resection of the retromuscular orbicularis oculi tissue to eliminate bulkiness of the lateral eyelid and eyebrow has been mentioned by Owsley [5], May et al. [6], and many others.

Although both procedures are referred to as upper blepharoplasty in the Western literature, the double eyelid operation and upper blepharoplasty are similar, but not identical. The double eyelid operation, a misnomer for blepharoplasty for young Asians, is a procedure for obtaining aesthetically pleasing upper eyelids in younger patients by creating a fold and thinning the eyelids. In contrast, blepharoplasty is a procedure used for obtaining youthful eyelids by excising sagging tissues. The difference between the double eyelid operation and senile blepharoplasty mainly relates to the degenerative changes in the tissue that are more prevalent in patients who undergo the latter procedure.
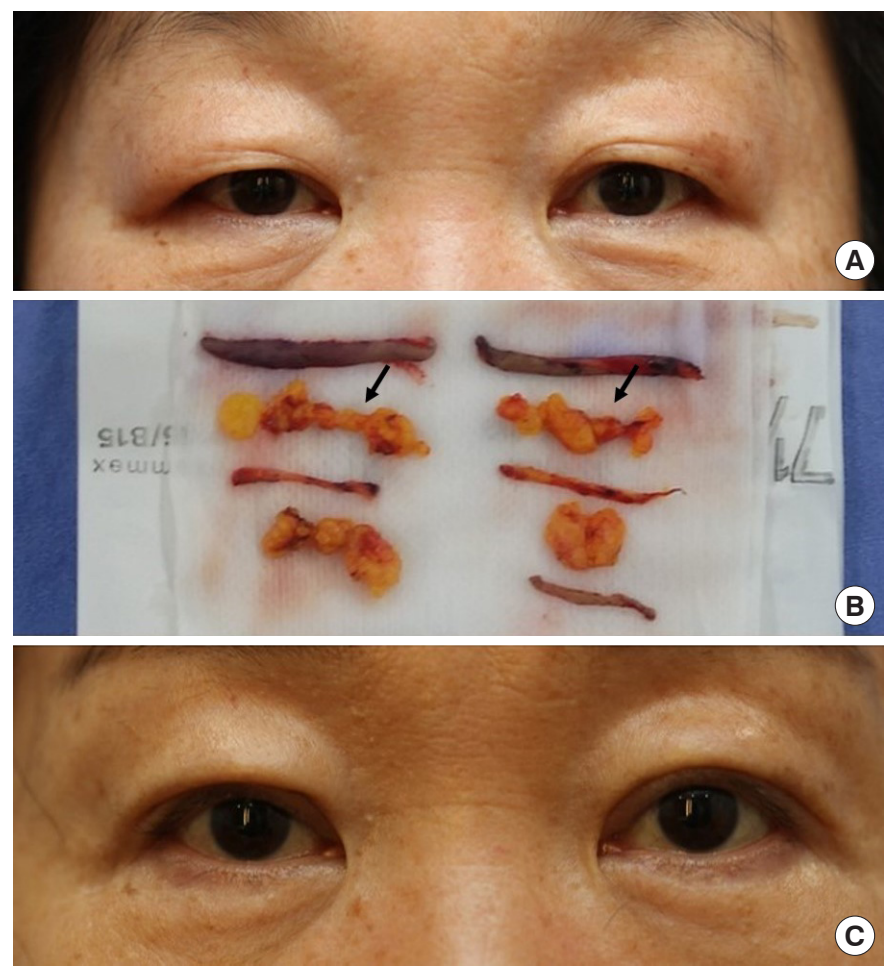

Fig. 8. Case of a 54-year-old female patient. (A) Preoperative photo. (B) Resected tissue; the black arrow points to the retromuscular fat. (C) Postoperative photo taken at 1 month after revision.

In blepharoplasty in Asian patients, a strip of orbicularis muscle and orbital fat is often resected for de-bulking of the upper eyelid $[7,8]$. Yoon and Park [1] classified the resectable tissues of the upper eyelids of young individuals and resected the tissues selectively according to the thickness of the eyelids. Ichinose and Tahara [9] also performed extended preseptal fat resection, including retroorbicularis oculi fat resection, in Asian patients. The aesthetic goal of the double eyelid operation is to turn a puffy eyelid into a thin eyelid with natural-appearing folds. However, the postoperative occurrence of extra folds and hollowness is more common in patients with very severe dermatochalasis than in young individuals after blepharoplasty due to the inelasticity and loss of pliability caused by senile degenerative changes associated with dermatochalasis. Many surgeons treat the undesired occurrence of sunken eyelids due to excessive removal of soft tissue during primary blepharoplasty. We agree with Rohrich et al. [10] that methods of periorbital rejuvenation are more conservative, are based on a concise preoperative evaluation, and involve the limited resection of the coveted soft tissue from the eye to restore a youthful appearance. Therefore, we used a conservative strategy while resecting the remnant skin, retromuscular tissue, and orbital fat during senile blepharoplasty. We limited retromuscular fibro-fatty tissue resection to the area within $7-10 \mathrm{~mm}$ from the upper incision in the central and lateral regions to create thin, natural-looking folds without thinning the 
entire eyelid.

We do not consider limited retromuscular tissue resection to be a unique method of obtaining natural-looking folds. However, we advocate for the use of this method in cases of senile Asian blepharoplasty as it allows more natural-appearing results to be obtained. Retromuscular tissue resection was deemed to be appropriate in $75 \%$ of the patients treated at our clinic, implying that the potential need for limited retromuscular tissue resection should not be ignored.

Evaluation of the upper eyelid must include an evaluation of the eyebrow. Brow ptosis should be corrected to achieve repositioning of the heavy eyebrow skin, which may be compensated for by contraction of the frontalis muscle to keep the eyebrows above the orbital rim. The patients in our study chose not to undergo the brow procedure because their insurance did not include it under coverage for blepharoptosis [11]. However, in cases of severe dermatochalasis or blepharoptosis, an eyelid incision is additionally needed for a brow lift.

Pretarsal tissue resection is indicated for young Asian patients to prevent pretarsal bulging after fold creation. Pretarsal tissue resection is not recommended for patients with senile blepharoptosis, as it leads to a longer period of edema and increases morbidity due to degenerative changes in the lymphatic drainage.

Our method of retromuscular tissue resection during senile blepharoplasty was confined to the central and lateral eyelid and to the area within $10 \mathrm{~mm}$ of the incision margin. We did not resect the retromuscular tissue in the nasal third of the upper eyelid, since the tissue in this area is already thin and is prone to folding in any direction. Furthermore, our technique ensured that a significant amount of central and lateral orbital fat was preserved, and the lower edge was included during skin closure to prevent hollowness and unwanted folds, which can occur due to adhesion of the levator and OOMs.

In senile blepharoplasty, a thick, unnatural-looking double eyelid fold can result from excessive skin excision. Therefore, in this study, the remnant skin was excised conservatively, and the distance from the upper incision line to the inferior border of the eyebrow was maintained at a minimum of $15 \mathrm{~mm}$ (Fig. 3).

To create a natural-looking fold, we induced appropriate adhesion by incorporating the posterior lamella of the eyelid (the aponeurosis) during skin closure. In contrast, procedures involving a buried suture between the aponeurosis and the dermis tend to create an artificial-looking fold.

In conclusion, senile blepharoplasty is a method of creating weak folds and is different from double eyelid surgery, as it involves limited retromuscular tissue resection without pretarsal exploration.

\section{NOTES}

\section{Conflict of interest}

No potential conflict of interest relevant to this article was reported.

\section{Ethical approval}

The study was approved by the Institutional Review Board of Kangbuk Samsung Hospital (IRB No. 2019-07-020) and performed in accordance with the principles of the Declaration of Helsinki.

\section{Patient consent}

The patients provided written informed consent for the publication and the use of their images.

\section{ORCID}

Seo Gil Choi

Sang Woo Suk

Kun Chul Yoon

https://orcid.org/0000-0002-8945-2278

https://orcid.org/0000-0002-7647-841X

https://orcid.org/0000-0001-9167-6716

\section{REFERENCES}

1. Yoon KC, Park S. Systematic approach and selective tissue removal in blepharoplasty for young Asians. Plast Reconstr Surg 1998;102:502-8.

2. Bourguet J. Notre traitement chirurgical de "poches" sous les yeux sans cicatrice. Arch Fr Belg Chir 1928;31:133.

3. Castanares S. Blepharoplasty for herniated intraorbital fat; anatomical basis for a new approach. Plast Reconstr Surg (1946) 1951;8:46-58.

4. Knize DM. An anatomically based study of the mechanism of eyebrow ptosis. Plast Reconstr Surg 1996;97:1321-33.

5. Owsley JQ. Resection of the prominent lateral fat pad during upper lid blepharoplasty. Plast Reconstr Surg 1980;66:165.

6. May JW Jr, Fearon J, Zingarelli P. Retro-orbicularis oculus fat (ROOF) resection in aesthetic blepharoplasty: a 6-year study in 63 patients. Plast Reconstr Surg 1990;86:682-9.

7. Flowers RS. Asian blepharoplasty. Aesthet Surg J 2002;22:558-68.

8. McCurdy JA Jr. Upper blepharoplasty in the Asian patient: the "double eyelid" operation. Facial Plast Surg Clin North Am 2002;10:351-68.

9. Ichinose A, Tahara S. Extended preseptal fat resection in Asian blepharoplasty. Ann Plast Surg 2008;60:121-6.

10. Rohrich RJ, Coberly DM, Fagien S, et al. Current concepts in aesthetic upper blepharoplasty. Plast Reconstr Surg 2004;113:32e-42e.

11. Flowers RS, Flowers SS. Precision planning in blepharoplasty: the importance of preoperative mapping. Clin Plast Surg 1993;20:303-10. 